\title{
Conocimiento de los factores de riesgo vocal y presencia de síntomas vocales en profesores universitarios
}

\section{Knowledge of vocal risk factors and presence of vocal symptoms in university professors}

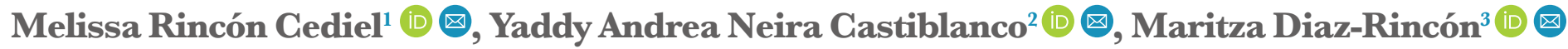 \\ ${ }^{1}$ Programa de Fonoaudiología; Universidad Manuela Beltrán; Bucaramanga; Colombia. \\ ${ }^{2}$ Escuela de Ingeniería de Producción; Instituto Universitario de la paz-UNIPAZ; Barrancabermeja; Colombia. \\ ${ }^{3}$ Facultad de Salud; Universidad del Sinú; Cartagena; Colombia.
}

Correspondencia

Melissa Rincón Cediel, email:

flga.melissarinconcediel@outlook.es

\begin{abstract}
Citar así
Rincón Cediel, Melissa; Neira Castiblanco, Yaddy Andrea; Diaz-Rincón, Maritza. (2021). Conocimiento de los factores de riesgo vocal y presencia de síntomas vocales en profesores universitarios. Revista de Investigación e Innovación en Ciencias de la Salud. 3(1), 22-32. https://doi. org/10.46634/riics.59
\end{abstract}

Recibido: 13/01/2021

Revisado: $05 / 02 / 202$

Aceptado: 15/02/2021

Editora invitada

Lady Catherine Cantor-Cutiva, Ph.D. (iD

Editor in chief

Jorge Mauricio Cuartas Arias, Ph.D. (iD

Goeditor

Fraidy-Alonso Alzate-Pamplona, MSc. (iD

Gopyright $($ ) 2021. Fundación Universitaria

María Cano. La Revista de Investigación e

Innovación en Ciencias de la Salud proporciona acceso abierto a todo su contenido bajo los términos de la licencia Creative Commons Attribution-NonCommercial-NoDerivatives 4.0 International (CG BY-NG-ND 4.0).

Declaración de intereses

Los autores han declarado que no hay conflicto de intereses.

\section{Resumen}

Introducción: existen diferentes factores de riesgo que pueden llegar a generar alteraciones vocales en los profesores en el ejercicio de su labor. Esta investigación busca establecer la relación entre el conocimiento de los factores de riesgo vocal y la presencia de síntomas asociados a enfermedad vocal en profesores universitarios.

Método: se llevó a cabo un estudio observacional descriptivo con un diseño transversal con 200 profesores seleccionados bajo criterios de exclusión y exclusión. Se aplicó una encuesta online de conocimiento de los factores de riesgo vocal y la subescala física del Índice de Incapacidad Vocal (VHI). Para el análisis estadístico se usó Stata 14 .

Resultados: se encontró que los docentes presentan conocimiento de los factores de riesgo vocal y la presencia de síntomas es leve. La relación entre estas dos variables fue negativa, es decir, a mayor conocimiento vocal menor presencia de síntomas.

Conclusiones: a pesar de encontrarse relación entre las variables de estudio, el nivel de significancia es bajo. Las investigaciones que indagan el conocimiento de los factores de riesgo vocal son reducidas, por lo que se recomienda continuar investigando esta temática.

Palabras clave

Conocimiento; disfonía; docentes; factores de riesgo; voz; síntomas vocales; riesgo vocal; profesores universitarios; vocología.

\section{Abstract}

Introduction: there are different risk factors that can generate vocal alterations in teachers in the exercise of their work. This research seeks to establish the relationship between knowledge of vocal risk factors and the presence of symptoms associated with vocal disease in college teachers. 
Disponibilidad de datos

Todos los datos relevantes se encuentran en el artículo. Para mayor información, comunicarse con el autor de correspondencia.

\section{Financiamiento}

Ninguno. Esta investigación no recibió ninguna subvención específica de agencias de financiamiento en los sectores público, comercial o sin fines de lucro.

\section{Descargo de responsabilidad}

El contenido de este artículo es responsabilidad exclusiva de los autores y no representa una opinión oficial de sus instituciones ni de la Revista de Investigación e Innovación en Ciencias de la Salud.

\section{Agradecimientos}

A la Universidad Manuela Beltrán.
Method: a descriptive observational study was carried out with a cross-sectional design with 200 teachers selected under exclusion and exclusion criteria. An online survey of knowledge of vocal risk factors and the physical subscale of the Vocal Disability Index (VHI) were applied. Stata 14 was used for statistical analysis.

Results: it was found that teachers present knowledge of vocal risk factors and the presence of symptoms is mild. The relationship between these two variables was negative, that is, the higher the vocal knowledge, the lower the presence of symptoms.

Conclusions: despite finding a relationship between the study variables, the level of significance is low. The investigations that research the knowledge of the vocal risk factors are limited. Therefore, it is recommended to continue investigating this topic.

\section{Keywords}

Knowledge; dysphonia; faculty; risk factors; voice; vocal symptoms; vocal risk; University teachers; vocology.

\section{Introducción}

Los profesionales de la voz deben conocer las fortalezas y debilidades que puede tener su voz, así como las amenazas externas que aumentan la probabilidad de padecer un desorden vocal, impidiéndoles llevar a cabo el objetivo comunicativo que se persigue. En el caso de los profesores, el objetivo comunicativo puede ser captar la atención de los estudiantes, convencerlos, motivarlos, etc. [1-2].

Pasa et al. [3] concluyeron que un programa de higiene vocal es efectivo, pues incrementa el conocimiento de la voz y reduce los síntomas vocales. Pedersen et al. [4] demostraron que un programa de higiene de voz, incluyendo la respiración abdominal durante el habla, además de un cambio en el patrón de entonación y el entrenamiento hacia una voz resonante, podrían mejorar el comportamiento vocal. Van Houtte et al. [5] investigaron el conocimiento frente al cuidado vocal, la búsqueda de ayuda médica y el ausentismo laboral por problemas de voz en 994 profesores, encontrando que el $13,5 \%$ tenía conocimiento del cuidado vocal, el 25,4\% buscaron ayuda médica y el 20,6\% había perdido al menos un día de trabajo por enfermedad vocal.

El riesgo vocal es la posibilidad de desarrollar un deterioro de la voz asociado a diferentes factores [6], entre los que se encuentran: edad, género, tipo de labor desarrollada, personalidad, factores ambientales (aire seco, cambios de temperatura significativos, ambientes acústicos con condiciones reverberantes), factores relacionados con el estilo de vida (tabaquismo, deshidratación), problemas médicos (asma, reflujo gastroesofágico, alergias respiratorias) [7], entre otros.

Existen ocupaciones que tienen mayor probabilidad de desarrollar trastornos de la voz, debido a que hacen un mayor uso de ella, en contraste, con otras labores. Este es el caso de profesores, locutores, telefonistas, sacerdotes, abogados, etc. Estudios realizados muestran un predominio de desórdenes de la voz en profesores [5,8,9], producto de una demanda excesiva de uso vocal, falta de conocimiento en el adecuado uso de la voz, inapropiadas condiciones ambientales de las aulas y pocas pausas de descanso [10]. Kyriacou et al. [11], en un estudio realizado con 194 profesores universitarios, encontraron que el tiempo de uso vocal, los hábitos y los factores del entorno de las aulas contribuyen al desarrollo de los desórdenes vocales. Korn et al. 
[12] hallaron un alto porcentaje de ronquera en profesores universitarios, con predominio mujeres, y factores asociados como el ruido ambiental y la competencia vocal con el ruido.

También se evidencia el alto riesgo vocal en otras ocupaciones que usan la voz como principal herramienta de trabajo, este es el caso de los predicadores [13], y el riesgo se asocia a la poca información recibida en el cuidado de la voz. Por otra parte, Ravall et al. [14], en un estudio donde participaron 315 cantantes de coro, identificaron un pobre conocimiento en anatomía, fisiología y ergonomía de la voz; también detectaron que el 21\% de ellos presentaba síntomas vocales. En ese estudio se concluyó que es importante educar en el cuidado vocal a los cantantes de coro para prevenir desórdenes vocales.

La disfonía puede manifestarse con la presencia de síntomas auditivos y/o propioceptivos, como cansancio al hablar, carraspeo, ronquera, ardor en la garganta, disminución del volumen vocal, entre otros [15]. Las escalas de Autorreporte vocal se usan en la clínica vocal para conocer la percepción de la persona frente a su propia voz, teniendo en cuenta aspectos sociales, funcionales y psicológicos, y también para establecer el progreso ante la terapia vocal (directa y/o indirecta). En el ámbito de la salud en el trabajo, se convierten en una herramienta útil para la detección de síntomas vocales [16], lo que contribuye a la toma de decisiones frente a las actividades de conservación de la salud vocal.

La disfonía es considerada como enfermedad laboral en Colombia [17]. Dentro de las ocupaciones con probabilidad de padecerla, se encuentran los docentes, debido a un esfuerzo vocal constante. Así mismo, la Organización Internacional del Trabajo (OIT) ha identificado a los profesores como un grupo con alta susceptibilidad a presentar alteraciones vocales por su labor [18]. El presente estudio tiene como objetivo establecer si existe una asociación entre el conocimiento del riesgo vocal y los síntomas vocales en docentes universitarios.

\section{Metodología}

Se llevó a cabo un estudio observacional descriptivo de corte transversal, en docentes universitarios de una institución de Barrancabermeja, Colombia. El tamaño de la muestra fue calculado en el programa Epidat 3,1 de la OPS/OMS, teniendo en cuenta como parámetros una significancia estadística de 0,05 y error del $5 \%$. Adicionalmente, se calculó un porcentaje de falta de participación del 8\%, con lo cual se estimó una muestra final de 200 docentes. Los individuos fueron seleccionados mediante un muestreo no probabilístico por conveniencia, en el que se incluyeron aquellos vinculados con modalidad de tiempo completo, medio tiempo y hora cátedra de las escuelas de Ciencias, Ingeniería de Producción, Ingeniería Ambiental y Saneamiento, Ingeniería Agronómica, Ingeniería Agroindustrial y Medicina Veterinaria y Zootecnia. Se excluyeron docentes investigadores y/o con funciones netamente administrativas y aquellos con experiencia docente inferior a 6 meses y/o con diagnóstico previo o actual de enfermedad vocal reportada en los exámenes médicos ocupacionales.

La base de datos fue manejada exclusivamente por los investigadores, lo que garantiza la confidencialidad y privacidad de la información. Antes de la aplicación del instrumento, los docentes firmaron el consentimiento informado de manera física. Este estudio fue aprobado por el Comité de Ética de la Universidad Manuela Beltrán con Acta No.3-18 del 24 de abril de 2018 y con número de radicación MSST 18101. 


\section{Recolección de información}

Se diseñó un instrumento con 7 secciones y un total de 48 preguntas. La primera sección, conformada por 3 preguntas, se estableció para la toma de datos generales de los docentes. La segunda incluyó 5 preguntas categóricas de información sociodemográfica: sexo, edad, estado civil, profesión y nivel educativo. La tercera sección, compuesta por 6 preguntas categóricas y cuantitativas, recolectó información ocupacional: tiempo de experiencia (en general) en la labor docente, experiencia en la institución universitaria, tipo de vinculación, horas de docencia directa a la semana con la institución universitaria y en otras instituciones, número de clases en bloque (de 3 o más horas seguidas). La cuarta sección, conformada por 4 preguntas dicotómicas (SI-NO) acerca del posible origen del conocimiento del riesgo vocal, indagó por las capacitaciones recibidas en salud vocal, la realización de terapia vocal y/o entrenamiento vocal previo y la consulta por cuenta propia acerca del cuidado de la voz. La quinta sección, constituida por 5 preguntas dicotómicas (SI-NO), recogió información sobre los factores médicos asociados al riesgo vocal. En ese sentido, se indaga por la existencia de diagnóstico médico previo de enfermedad: por reflujo gastroesofágico, de tipo hormonal, respiratorio, aclaración de consumo de medicamentos y mención de cuáles (para establecer si eran causantes de síntomas vocales por deshidratación) [19] y la realización de otro tipo de actividad extralaboral que implique una demanda vocal alta. La sexta sección estaba conformada por 15 preguntas de selección múltiple que buscaban identificar el conocimiento de los docentes sobre los factores de riesgo vocal. Esta sección se dividió en tres dimensiones con 5 preguntas cada una: el nivel de conocimiento de los factores laborales, la higiene y el entrenamiento vocal.

Para la variable de conocimiento sobre riesgo vocal se generaron las siguientes categorías: categoría 1 (0-5 respuestas correctas); categoría 2 (6-10 respuestas correctas); categoría 3 (1115 respuestas correctas). Por último, la séptima sección recolectó información relacionada con la presencia de síntomas asociados a la voz en los docentes. Las preguntas fueron extraídas de la subsección II (escala física) del test adaptado y validado del Índice de Incapacidad Vocal (VHI-30) [20]. Este apartado consta de diez ítems con opciones de respuesta tipo Likert (0 nunca, 1 casi nunca, 2 algunas veces, 3 casi siempre y 4 siempre). Se evaluó teniendo en cuenta la sumatoria de las respuestas y se realizó la clasificación de la presencia de síntomas según lo establecido en la escala: leve $<20$ puntos, moderada $21-30$ puntos y severa $>30$ puntos.

Posterior al diseño del cuestionario se llevó a cabo una revisión por expertos en la que participaron 7 jueces: tres fonoaudiólogos con formación y experiencia posgradual en el área de voz, tres profesionales de salud con formación y experiencia posgradual en seguridad y salud en el trabajo y un profesional en lingüística. Los expertos evaluaron suficiencia, claridad, coherencia y relevancia de cada uno de los ítems. A partir de allí se realizó un análisis cualitativo de los juicios y se procedió a hacer los ajustes al instrumento cuando había acuerdo entre 3 o más jueces. Además, se realizó el pilotaje del instrumento con una muestra del 10\% de los docentes vinculados durante el semestre y se obtuvo un total de 35 encuestados en el proceso de pilotaje. El instrumento fue aplicado en línea, a través de los correos institucionales de los docentes.

\section{Análisis estadístico}

Se realizó un análisis descriptivo para obtener la frecuencia absoluta y relativa de las variables de interés. Posteriormente, se realizó la prueba no paramétrica de Kruskal-Wallis de una vía, para determinar si el nivel de conocimiento en los docentes era diferente en los grupos de riesgo vocal. Adicionalmente, se realizó correlación de Pearson entre el puntaje de conocimientos 
sobre riesgo vocal y el puntaje de la presencia de síntomas en la voz. Para todos los análisis, las asociaciones se consideraron estadísticamente significativas, con un alfa $<$ de 0,05. Los análisis se llevaron a cabo con Stata 14 (StataCorp LP, College Station, EE. UU.).

\section{Resultados}

La muestra analítica estuvo compuesta por 200 participantes mayores de 20 años, docentes de una institución universitaria de Barrancabermeja, Santander. Entre ellos, el 53\% eran de sexo masculino. Con respecto al estado civil, 65,09\% de los hombres afirmaron tener pareja y en las mujeres el 51,06\%. Respecto al nivel educativo, casi la mitad de los participantes tenían estudios de pregrado. Esta proporción fue menor en hombres que en mujeres: 40,57\% y 56,38, respectivamente. El 26\% eran especialistas y el 25\% magíster.

Con relación a las características laborales, la mayoría de los participantes estaban adscritos a la escuela de Ciencias (61\%) y el 61,5\% tenían menos de 8 años de experiencia en docencia. La Tabla 1 expone las características sociodemográficas, laborales y de salud de los participantes según el sexo. La prevalencia de incapacidad vocal leve fue de 95\%, de incapacidad moderada el 4\% y solo el 1\% con incapacidad severa, de acuerdo con el índice de incapacidad vocal (VHI). Los 2 casos de incapacidad vocal severa se presentaron en mujeres, entre 31-40 años y con 9-15 años de antigüedad como docente. Respecto al conocimiento de los factores de riesgo vocal, el 72,50\% respondieron de manera correcta entre 6 - 10 preguntas; $27,50 \%$, entre $11-15$ preguntas; y el $0.5 \%$, entre $1-5$ preguntas. De aquellos que respondieron entre 6-10 preguntas adecuadamente, el 94,48\% presentaban un nivel de incapacidad vocal leve. Por otra parte, se encontró que gran parte del conocimiento de los docentes sobre el riesgo vocal provenía de información disponible en internet, seguido de la información suministrada por expertos en el tema.

Con respecto al análisis bivariado, no se encontró una diferencia estadísticamente significativa entre la mediana del puntaje de riesgo vocal en los tres grupos de comparación, según lo estimado en la prueba no paramétrica Kruskal-Wallis ( $\mathrm{p}>0,05)$. Finalmente, se encontró una tendencia a la correlación negativa baja $(\mathrm{r}=-0,069)$. No obstante, esta correlación no fue estadísticamente significativa (Figura 1).

\section{Discusión}

Los participantes del estudio mostraron conocimiento de los factores de riesgo vocal y la presencia de síntomas fue leve. Según los hallazgos, el conocimiento frente a los factores de riesgo vocal lo obtuvieron de capacitaciones o búsqueda de información por cuenta propia. Igualmente, algunos han recibido terapia y/o entrenamiento vocal. El conocimiento de los factores de riesgo vocal contribuye a la salud vocal, en la medida que se apliquen los consejos dados. Bolbol et al. [21] llevaron a cabo un estudio comparativo donde se buscaba aumentar la conciencia en profesores frente a la higiene vocal, proporcionando información durante 3 meses acerca de la anatomía y fisiología vocal, los factores de riesgo, los síntomas de fatiga vocal y las pautas de cuidado vocal (hidratación, sueño, alimentación saludable, descanso vocal después del trabajo, evitar gritar, hablar con ruido de fondo, etc.), lo que mejoró considerablemente la actitud frente al cuidado vocal, expresándose en su salud vocal. Por otro lado, la prevalencia de los desórdenes de voz es mayor en mujeres [5,22]. En el grupo de estudio se observó un predominio de hombres. Sin embargo, fueron mujeres las que presentaron una puntuación moderada y severa para síntomas vocales, y el 70\% manifiesta no haber recibido capacitaciones en el cuidado vocal. Por otro lado, más de la mitad de los profesores no superaban los 


\section{Tabla 1. Características sociodemográficas, laborales y de salud, por sexo en} docentes de una Institución Universitaria de Barrancabermeja, Colombia

\section{Variables}

Todos $(\mathbf{n = 2 0 0 )} \%$

Hombres $(n=106) \%$

Mujeres $(n=94) \%$

\section{Sociodemográficas}

Grupo de edad (años)

\begin{tabular}{c|c|c|c}
\hline $21-30$ & 21 & 15,09 & 27,66 \\
\hline $31-40$ & 35 & 27,36 & 43,62 \\
\hline $41-50$ & 28 & 33,96 & 21,28 \\
\hline $51-60$ & 13 & 18,87 & 7,45 \\
\hline 61 o más & 2 & 4,72 & 0 \\
\hline Tener pareja & 58,5 & 65,09 & 51,06 \\
\hline
\end{tabular}

Nivel Educativo

\begin{tabular}{l|l|l|l}
\hline Pregrado & 48 & 40,57 & 56,38 \\
\hline Especialización & 26 & 33,02 & 19,15 \\
\hline Maestría & 25 & 26,42 & 24,47 \\
\hline
\end{tabular}

Laborales

Escuela adscrita

\begin{tabular}{l|c|c|c}
\hline Ciencias & 61 & 62 & 60 \\
\hline Ing. Agro-industrial & 5 & 3 & 7 \\
\hline Ing. Agronómica & 3 & 5 & 2 \\
\hline Ingeniería Ambiental & 4 & 5 & 4 \\
\hline Ing. de Producción & 17,5 & 18 & 17 \\
\hline Medicina Veterinaria & 8,5 & 13 & 4 \\
\hline
\end{tabular}

Tiempo en docencia (años)

\begin{tabular}{c|c|c|c}
\hline $0,5-8$ & 61,5 & 52,83 & 71,28 \\
\hline $9-15$ & 21 & 19,81 & 22,34 \\
\hline $16-25$ & 8,5 & 13,21 & 3,19 \\
\hline$>=26$ & 9 & 14,15 & 3,19 \\
\hline
\end{tabular}

Tipo de vinculación laboral

\begin{tabular}{l|c|c|c}
\hline \multicolumn{1}{c|}{ Hora Cátedra } & 24,5 & 29,25 & 19,15 \\
\hline \multicolumn{1}{c|}{ Medio tiempo } & 7,5 & 7,55 & 7,45 \\
\hline \multicolumn{1}{c|}{ Tiempo completo } & 68 & 63,21 & 73,4 \\
\hline Capacitaciones cuidado vocal (si) & 66 & 40 & 26 \\
\hline $\begin{array}{l}\text { Búsqueda propia de información sobre cui- } \\
\text { dado de la voz (si) }\end{array}$ & 109 & 61 & 48 \\
\hline Ha recibido terapia vocal (si) & 20 & 13 & 7 \\
\hline Entrenamiento en técnica vocal (si) & 36 & 30 & 6 \\
\hline
\end{tabular}

Presencia de síntomas

\begin{tabular}{l|c|c|c}
\hline Leve & 95 & 98,11 & 91,49 \\
\hline Moderada & 4 & 1,89 & 6,38 \\
\hline Severa & 1 & 0 & 2,13 \\
\hline
\end{tabular}


Factores de riesgo y síntomas vocales

Rincón Cediel et al.

Conocimiento sobre riesgo vocal

\begin{tabular}{l|c|c|c}
\hline \multicolumn{1}{c|}{ Categoría 1 (0-5 respuestas correctas) } & 0,5 & 0 & 1,06 \\
\hline \multicolumn{1}{c|}{ Categoría 2 (6-10 respuestas correctas) } & 72,5 & 75.47 & 69,15 \\
\hline \multicolumn{1}{c|}{ Categoría 3 (11-15 respuestas correctas) } & 27,5 & 24,53 & 29,79 \\
\hline Condiciones de salud & 19 & 7 & 12 \\
\hline Diagnóstico de reflujo & 18 & 1 & 17 \\
\hline Diagnóstico de enfermedad hormonal & 13 & 5 & 8 \\
\hline Diagnóstico enfermedad respiratoria &
\end{tabular}

Nota. Los resultados se presentan por columnas.

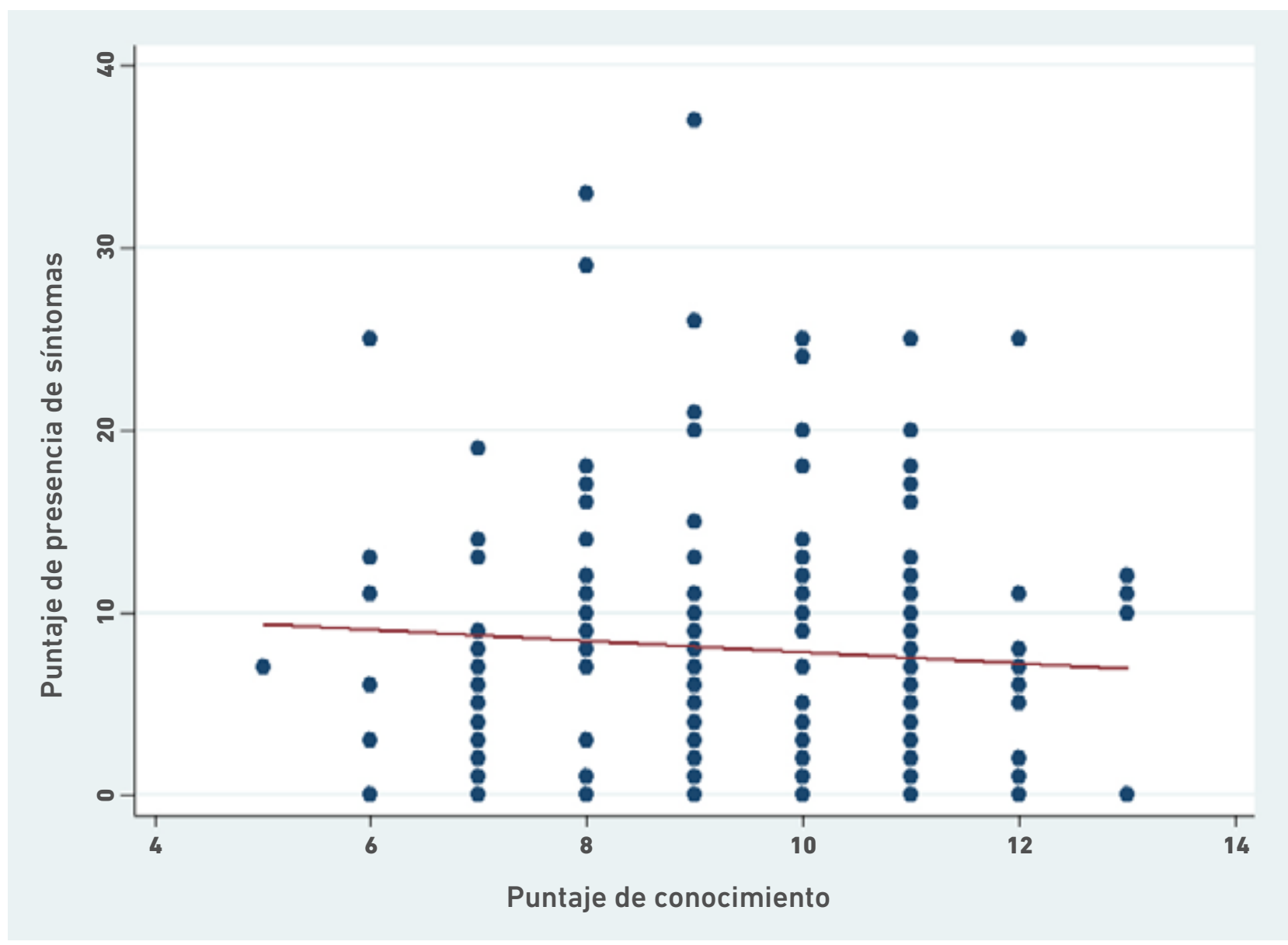

Figura 1. Correlación entre la presencia de síntomas vocales y el puntaje de riesgo vocal 
50 años. La edad es otro factor de riesgo para la presencia de trastornos de la voz, teniendo en cuenta que los cambios que ocurren con la edad se ven reflejados en la voz, apareciendo presbifonia. Gois et al. [23] encontraron una prevalencia de desórdenes de voz en mujeres y mayores de 60 años. También, Roy et al. [24] concluyeron que ser mujer, entre los 40 y los 59 años, y tener 16 años o más laborando, está asociado con los problemas de voz. En nuestro estudio la mayoría de los profesores tenían entre 0,5 y 8 años en la docencia.

Así mismo, existen otros factores de riesgo para la presencia de síntomas vocales, como lo son el reflujo y los problemas respiratorios [10]. En nuestro estudio, un grupo muy pequeño de la población presentaba diagnóstico de estas enfermedades. Martins et al. [25] evaluaron 2019 pacientes de diferentes edades para establecer el origen de la disfonía, encontrando un gran número de ellos con reflujo gastroesofágico y enfermedades nasosinusales. De esta manera, los síntomas de la disfonía pueden estar asociados a varios factores, que deben ser abordados de manera particular; el conocimiento de los factores de riesgo por sí solo no supone una medida preventiva.

Por otra parte, Señaris González et al. [26] evaluaron los factores que pueden influir en la percepción de la disfonía por parte del paciente, cuantificada mediante el VHI, encontrando que el VHI tiene relación con el parámetro acústico armónico/ruido (HNR) y con los parámetros B y S de la escala GRBAS. Las escalas funcional y emocional son las que más contribuyen a dicha relación; en nuestro estudio sólo se tuvo en cuenta la subescala física (síntomas), lo que pudo haber limitado la percepción que los profesores tienen frente a los posibles síntomas de la disfonía. Igualmente, aunque el Indice de Incapacidad Vocal (VHI) es útil como autorreporte de síntomas, es mucho más significativo cuando existe disfonía. Dentro de los criterios de exclusión no se tuvieron en cuenta los profesores con patología vocal. Si bien los profesores en el ejercicio de su labor experimentan síntomas, pueden estar más asociados a la fatiga vocal $[27,28]$ en ausencia de patología vocal. El Índice de Fatiga Vocal (VFI), recientemente traducido y adaptado al español [29], mostró una buena consistencia interna y alta fiabilidad en los tres factores que evalúa: cansancio de la voz y evitación de uso vocal, malestar físico asociado con el uso vocal y mejora de los síntomas vocales con el descanso. Consta de 19 preguntas que amplían los posibles síntomas asociados a la fatiga vocal por el uso de la voz, que es la realidad experimentada por los profesores. Muchos de ellos manifiestan síntomas mientras usan la voz, que menguan con el descanso.

\section{Limitaciones y recomendaciones}

Este estudio cuenta con algunas limitaciones. Por ser un estudio de corte transversal, no permite establecer asociaciones causales entre la variable dependiente de interés y las variables independiente. Así mismo, la no aleatorización de la muestra, dado que fue un muestreo a conveniencia, puede sesgar en cierta medida los resultados hallados. Por lo tanto, se recomienda realizar estudios longitudinales y análisis multivariables que permitan establecer la relación no confundida entre las variables de interés analizadas. También es necesario realizar estudios que estén orientados en el diseño y validación de escalas que permitan determinar el nivel de conocimiento vocal. Finalmente, se recomienda continuar estudiando esta temática, abordando también las actitudes y prácticas frente al riesgo vocal. 


\section{Conclusiones}

A partir de los hallazgos obtenidos en el presente estudio, se concluye la existencia de una tendencia a la correlación negativa baja entre el conocimiento sobre los factores de riesgo vocal y la presencia de síntomas vocales en los docentes universitarios evaluados. No obstante, a pesar de que estos resultados no lograron obtener una significancia estadística, la docencia supone una alta demanda vocal. Siendo así, es importante que las instituciones educativas incluyan Programas de Vigilancia Epidemiológica para el control de los Desórdenes Vocales en sus Sistemas de Gestión en Seguridad y Salud en el Trabajo, que faciliten el conocimiento a los docentes acerca de los factores de riesgo vocal que pueden presentar y se empiece a generar en ellos una conciencia sobre las posibilidades y limitaciones de la propia voz. El sólo conocimiento no garantiza una reducción de síntomas, pues se requiere un cambio en la conducta vocal.

\section{Referencias}

1. Bustos Sánchez I. La voz: Técnica y Expresión. 2da Edición. Zaragoza: Paidotribo. 2013.

2. Merlin Servilha EA, Fialho da Costa AT. Knowledge about voice and the importance of voice as an educational resource in the perspective of university professors. Rev. CEFAC. 2015;17(1):13-26. DOI: https://doi.org/10.1590/1982-0216201514813

3. Pasa G, Oates J, Dacakis G. The relative effectiveness of vocal hygiene training and vocal function exercises in preventing voice disorders in primary school teachers. Logoped Phoniatr Vocol. 2007;32(3):128-40. DOI: https://doi.org/10.1080/14015430701207774

4. Pedersen M, Beranova A, Møller S. Dysphonia: medical treatment and a medical voice hygiene advice approach. A prospective randomised pilot study. Eur Arch Otorhinolaryngol. 2004 Jul;261(6):3 12-5. DOI: https://doi.org/10.1007/s00405-003-0641-8

5. Van Houtte E, Claeys S, Wuyts F, Van Lierde K. The impact of voice disorders among teachers: Vocal complaints, treatment-seeking behavior, knowledge of vocal care, and voice-related absenteeism. Journal of Voice. 2011;25(5):570-5. DOI: http://dx.doi.org/10.1016/j.jvoice.2010.04.008

6. Lobo BPL, Madazio GMV, Badaró FAR, Behlau MS. Vocal risk in preachers: Talkativeness, vocal loudness, and knowledge about vocal health and hygiene. Codas. 2018;30(2):27. DOI: http://dx.doi.org/10.1590/2317-1782/20182017089

7. Sanssené C, Bardi J, Welby-Gieusse M. Prevalence and Risk Factors of Voice Disorders in French Tour Guides. Journal of Voice. 2020;34(6):911-7. DOI: http://dx.doi. org/10.1016/j.jvoice.2019.05.002

8. Behlau M, Zambon F, Guerrieri AC, Roy N. Epidemiology of voice disorders in teachers and nonteachers in Brazil: Prevalence and adverse effects. Journal of Voice. 2012;26(5): 665.e9-665.e18. DOI: http://dx.doi.org/10.1016/j.jvoice.2011.09.010

9. Silva BG, Chammas TV, Zenari MS, Moreira RR, Samelli AG, Nemr K. Analysis of possible factors of vocal interference during the teaching activity. Rev Saude Publica. 2017; 51:124. DOI: http://dx.doi.org/10.11606/s1518-8787.2017051000092 
10. da Rocha LM, de Lima Bach S, do Amaral PL, Behlau M, de Mattos Souza LD. Risk Factors for the Incidence of Perceived Voice Disorders in Elementary and Middle School Teachers. Journal of Voice. 2017;31(2):258.e7-258.e12. DOI: http://dx.doi.org/10.1016/j.jvoice.2016.05.018

11. Kyriakou K, Petinou K, Phinikettos I. Risk Factors for Voice Disorders in University Professors in Cyprus. Journal of Voice. 2018;32(5):643.e1-643.e9. DOI: http://dx.doi. org/10.1016/j.jvoice.2017.07.005

12. Korn GP, Augusto De Lima Pontes A, Abranches D, Augusto De Lima Pontes P. Hoarseness and Risk Factors in University Teachers. Journal of Voice. 2015;29(4):518. e21-518. e28. DOI: http://dx.doi.org/10.1016/j.jvoice.2014.09.008

13. Hagelberg A, Simberg S. Prevalence of Voice Problems in Priests and Some Risk Factors Contributing to Them. Journal of Voice. 2015;29(3):389.e11-389.e18. DOI: http://dx. doi.org/10.1016/j.jvoice.2014.08.015

14. Ravall S, Simberg S. Voice Disorders and Voice Knowledge in Choir Singers. Journal of Voice. 2020;34(1):157.e1-157.e8. DOI: https://doi.org/10.1016/j.jvoice.2018.07.00515.

15. Gomes VEFI, Batista DDC, Lopes LW, Aquino R, Almeida AA. Symptoms and Vocal Risk Factors in Individuals with High and Low Anxiety. Folia Phoniatrica et Logopaedica. 2019;71(1):7-15. DOI: http://dx.doi.org/10.1159/000494211

16. Behlau M, Madazio G, Moreti F, Oliveira G, dos Santos L de MA, Paulinelli BR, et al. Efficiency and Cutoff Values of Self-Assessment Instruments on the Impact of a Voice Problem. Journal of Voice. 2016;30(4):506.e9-506.e18. DOI: http://dx.doi.org/10.1016/j.jvoice.2015.05.022

17. República de Colombia. Decreto 1477 (5 de agosto de 2014). Por el cual se expide la tabla de enfermedades laborales. Departamento Administrativo de la Función Pública. Disponible en: https://www.funcionpublica.gov.co/eva/gestornormativo/norma_pdf. php?i=58849

18. Ma EP, Yiu EM. Voice Activity and Participation Profile: Assessing the Impact of Voice Disorders on Daily Activities. J Speech Lang Hear Rev. 2016;511-24. DOI: http://dx. doi.org/10.1044/1092-4388(2001/040).

19. Núñez F, Mate MA. Historia clínica y valoración subjetiva de la voz (calidad de vida en relación con la voz). En: Cobeta I, Núñez F, Fernández S, coordinadores. Patología de la voz. Valencia: Marge Médica Books; 2013. p. 111-118. Disponible en: https://cutt. ly/snzSxrs

20. Núñez-Batalla F, Corte-Santos P, Señaris-González B, Llorente-Pendás JL, Górriz-Gil C, Suárez-Nieto C. Adaptación y validación del índice de incapacidad vocal (VHI-30) y su versión abreviada (VHI-10) al español. Acta Otorrinolaringol Española. 2007;58(9):38692. DOI: http://dx.doi.org/10.1016/S0001-6519(07)74954-3

21. Bolbol SA, Zalat MM, Hammam RAM, Elnakeb NL. Risk Factors of Voice Disorders and Impact of Vocal Hygiene Awareness Program Among Teachers in Public Schools in Egypt. Journal of Voice. 2017;31(2):251.e9-251.e16. DOI: http://dx.doi.org/10.1016/j. jvoice.2016.07.010 
22. Kim KH, Kim RB, Hwang DU, Won SJ, Woo SH. Prevalence of and Sociodemographic Factors Related to Voice Disorders in South Korea. Journal of Voice. 2016;30(2):246.e1246.e7. DOI: http://dx.doi.org/10.1016/j.jvoice.2015.04.010

23. Góis ACB, Pernambuco L, de Lima KC. Prevalence and Associated Factors With Voice Disorders in Brazilian Community-dwelling Older Adults. Journal of Voice. 2019;33(5):806.e1-806.e7. DOI: https://doi.org/10.1016/j.jvoice.2018.02.025

24. Roy N, Merril RM, Thibeault S, Parsa RA, Gray SD, Smith EM. Prevalence of Voice Disorders in Teachers and The General Population. Journal of Speech, Language, and Hearing Research. 2004;47:281-293. DOI: https://doi.org/10.1044/1092-4388(2004/023)

25. Martins RHG, do Amaral HA, Tavares ELM, Martins MG, Gonçalves TM, Dias NH. Voice Disorders: Etiology and Diagnosis. Journal of Voice [Internet]. 2016;30(6):761.e1761.e9. DOI: http://dx.doi.org/10.1016/j.jvoice.2015.09.017

26. Señaris González B, Núñez Batalla F, Corte Santos P, Suárez Nieto C. Índice de Incapacidad Vocal: factores predictivos. Acta Otorrinolaringológica Española. 2006;57(2):1018. DOI: https://doi.org/10.1016/S0001-6519(06)78670-8

27. Cantor-Cutiva LC, Banks R, Berardi M, Johnson B, Clawson R, Martinez S, Hunter E. From vocal effort to vocal fatigue. What does the literature say? Paper presented at the 11 th International Conference on Voice Physiology and Biomechanics; 2018; Michigan State University, East Lansing, MI, United States.

28. Hunter E, Cantor-Cutiva LC, van Leer E, van Mersbergen M, Nanjundeswaran CD, Bottalico P, et al. Towards a consensus description of Vocal Effort, Vocal Load, Vocal Loading, and Vocal Fatigue. JSLHR. 2019;63(2):509-532. DOI: https://doi. org/10.1044/2019_JSLHR-19-00057

29. Cantor-Cutiva LC, Malebrán Bezerra de Mello CM, Hunter EJ. Translation and Adaptation of the Vocal Fatigue Index into Spanish. Revista de Investigación e Innovación en Ciencias de la Salud. 2020;2(2): 04-13 DOI: https://doi.org/10.46634/riics.29 